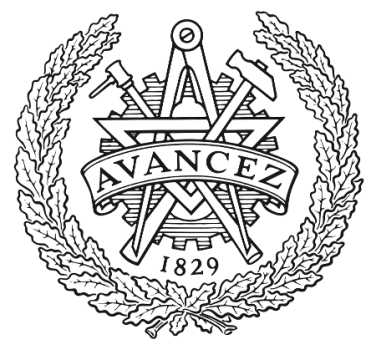

CHALMERS

UNIVERSITY OF TECHNOLOGY

\title{
FRET-Based Assay for the Quantification of Extracellular Vesicles and Other Vesicles of Complex Composition
}

Downloaded from: https://research.chalmers.se, 2023-04-26 13:39 UTC

Citation for the original published paper (version of record):

Thorsteinsson, K., Olsén, E., Schmidt, E. et al (2020). FRET-Based Assay for the Quantification of Extracellular Vesicles and Other Vesicles of Complex

Composition. Analytical Chemistry, 92(23): 15336-15343.

http://dx.doi.org/10.1021/acs.analchem.0c02271

N.B. When citing this work, cite the original published paper. 


\title{
FRET-Based Assay for the Quantification of Extracellular Vesicles and Other Vesicles of Complex Composition
}

\author{
Konrad Thorsteinsson, Erik Olsén, Eneas Schmidt, Hudson Pace, ${ }^{*, \perp}$ and Marta Bally*, \\ Cite This: Anal. Chem. 2020, 92, 15336-15343 \\ Read Online
}

ABSTRACT: Research in the field of extracellular vesicles is rapidly expanding and finding footholds in many areas of medical science. However, the availability of methodologies to quantify the concentration of membrane material present in a sample remains limited. Herein, we present a novel approach for the quantification of vesicle material, specifically the quantification of the total lipid membrane surface area, found in a sample using Förster resonance energy transfer (FRET). In this assay, sonication is used to drive the fusion between vesicles in the sample to be quantified and liposomes containing a pair of FRET fluorophores. The change in emission spectrum upon vesicle fusion is directly related to the total membrane surface area of the sample added, and a calibration curve

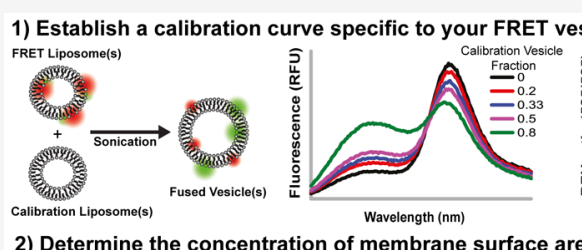

2) Determine the concentratic

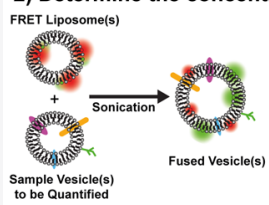

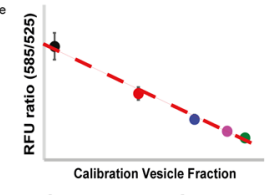
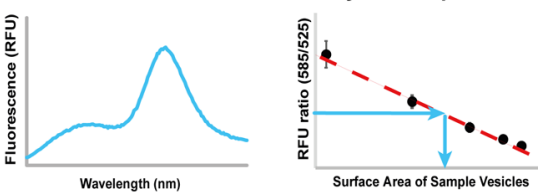
allows for the quantification of a variety of vesicle species, including enveloped viruses, bacterial outer membrane vesicles, and mammalian extracellular vesicles. Without extensive optimization of experimental parameters, we were able to quantify down to $\sim 10^{9}$ vesicles $/ \mathrm{mL}$, using as little as $60 \mu \mathrm{L}$ of the sample. The assay precision was comparable to that of a commercial nanoparticle tracking analysis system. While its limit of detection was slightly higher, the FRET assay is superior for the detection of small vesicles, as its performance is vesicle-size-independent. Taken together, the FRET assay is a simple, robust, and versatile method for the quantification of a variety of purified vesicle samples.

\section{INTRODUCTION}

Extracellular vesicles (EVs) produced by both eukaryotic and prokaryotic cells have been found to mediate intercellular communication in a variety of biological contexts. Within humans, EVs produced by our own cells have been shown, for example, not only to modulate immune response regulation, ${ }^{1-3}$ inflammation, ${ }^{4-6}$ or tissue regeneration ${ }^{7,8}$ but also to be involved in disease propagation. ${ }^{9-11}$ Bacterial EVs also contribute to modulating human physiology, both those produced by commensal bacteria in our digestive systems and those produced by pathogenic bacteria to propagate disease. ${ }^{12}$ Enveloped viruses represent yet another class of lipid bilayer-encapsulated biological particles, responsible for generating a variety of diseases in humans and other organisms. Liposomes are artificially produced vesicles that have been widely investigated as drug delivery vehicles. ${ }^{13-15}$ As outlined above, biological and synthetic vesicles are associated with a broad spectrum of research areas, all of which are currently hindered by the underdevelopment of methods to quantify lipid vesicle concentrations in a robust and versatile manner.

Traditionally, vesicle quantification has been performed using methods relying on the quantification of specific biomolecular constituents of the vesicles. Indeed, the concentration of an EV sample is often reported in terms of total protein content, given in protein mass per unit volume; ${ }^{16}$ for virus particles, the quantification of oligonucleotides can be used; ${ }^{17}$ while for liposomes, phosphorus quantification is an extremely robust approach to determine the number of phospholipids in the sample. A drawback of such approaches is that they only provide an indirect measure of the particle content, in particular, since the protein/particle or oligonucleotide/particle ratio is often unknown and may vary greatly among different vesicle types and sample batches. As a complement to biomolecular quantification approaches, methods allowing for the determination of particle concentrations, given in particles/volume, have been developed within the last decade. In particular, nanoparticle tracking analysis (NTA), ${ }^{18}$ which allows for the visualization of the light scattered by individual vesicles upon laser illumination, has gained in popularity for vesicle characterization. ${ }^{16,19-21}$ With this method, vesicle numbers can be quantified by particle counting and their size estimated upon tracking of their Brownian motion. A constraint of this method is that the relatively weak scattering properties of vesicles in solution limit

Received: May 27, 2020

Accepted: October 30, 2020

Published: November 12, 2020 
A

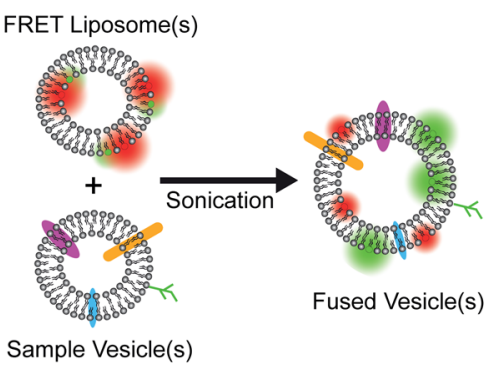

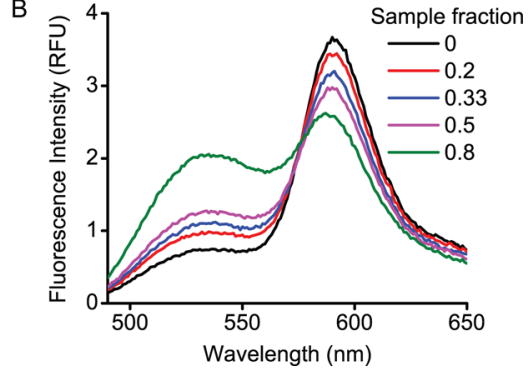

Figure 1. Schematic representation of the FRET assay. (A) FRET liposomes fuse with unlabeled sample vesicles to be quantified, thereby reducing the surface density of fluorophores. Fusion is facilitated by sonication. (B) Change in the emission spectrum of the fused vesicles as a function of sample vesicle percentage. Excitation wavelength: $460 \mathrm{~nm}$. The sample fraction is the ratio of sample vesicles in the sample.

the detection to those with diameters above $50-70 \mathrm{~nm} .{ }^{19}$ This is not sufficient to detect the smallest vesicle populations that may have characteristic diameters down to $30 \mathrm{~nm}^{22}$

Herein, we propose an alternative methodology for quantifying vesicle samples whose sensitivity is not dependent on vesicle size. Rather than using specific biomolecular targets (e.g., protein or phospholipids), our assay quantifies the total surface area (TSA) of membrane material (i.e., all membrane components) in the sample using a Förster resonance energy transfer (FRET)-based assay. In our assay design, inspired by liposome-based methods traditionally used to detect membrane fusion, ${ }^{23,24}$ sonication is used to drive the fusion of liposomes containing a pair of fluorophore-lipid conjugates subject to FRET with the sample vesicles to be quantified (Figure 1a). Upon fusion, the intermolecular distance of the FRET pair increases, resulting in a decrease in acceptor emission and an increase in donor emission (Figure 1B), whereby the change in the emission spectrum of the fused vesicles is directly related to the amount of sample vesicles added.

\section{MATERIALS AND METHODS}

Materials. All materials unless stated otherwise were purchased from commercial sources. Lipids used in this project were 1-palmitoyl-2-oleoyl-sn-glycero-3-phosphocholine (POPC), 1,2-dioleoyl-sn-glycero-3-phosphoethanolamine- $\mathrm{N}$ (lissamine rhodamine B sulfonyl) (Rhod-PE, Ex/Em: 560/ $583 \mathrm{~nm}$ ), and 1,2-dioleoyl-sn-glycero-3-phosphoethanolamine$\mathrm{N}$-(7-nitro-2-1,3-benzoxadiazol-4-yl) (NBD-PE, Ex/Em: 460/ $535 \mathrm{~nm}$ ). Chloroform was used as a solvent for all lipids. All lipids were obtained from Avanti Polar Lipids Inc. (Alabama). Chloroform, potassium antimonyl tartrate trihydrate $\left(\mathrm{C}_{8} \mathrm{H}_{4} \mathrm{~K}_{2} \mathrm{O}_{12} \mathrm{Sb}_{2} \cdot 3 \mathrm{H}_{2} \mathrm{O}\right)$, ammonium heptamolybdate tetrahydrate $\left(\left(\mathrm{NH}_{4}\right)_{6} \mathrm{Mo}_{7} \mathrm{O}_{24} \cdot 4 \mathrm{H}_{2} \mathrm{O}\right)$, and a phosphorus standard solution $(0.65 \mathrm{M})$ were obtained from Sigma-Aldrich (Darmstadt, Germany). 4-(2-Hydroxyethyl)-1-piperazineethanesulfonic acid (HEPES), sulfuric acid, and ascorbic acid were obtained from Merck (New Jersey), while phosphate-buffered saline (PBS) tablets were obtained from Medicago $A B$ (Uppsala, Sweden) and $\mathrm{NaCl}$ was obtained from VWR Chemicals (Leuven, Belgium). All water samples used in the experiments were deionized and filtered using a Milli-Q system (Millipore, France).

Outer membrane vesicles (OMVs) from an Escherichia coli mutant with truncated lipopolysaccharides (hldE strain (RN102)) were kindly provided by Madeleine Ramstedt (Department of Chemistry, Umeå University) and produced as previously described. ${ }^{25}$ Herpes simplex virus type 2 (HSV-2) of the strain 333 (wt-333) ${ }^{26}$ was produced by infecting GMK AH1 cells, followed by purification through a three-step discontinuous sucrose gradient as previously described. ${ }^{27,28}$ The virus was kindly provided by Tomas Bergström (Department of Infectious Diseases, University of Gothenburg). The eukaryotic extracellular vesicles, hereforth referred to as EVs, were produced by a rat oligodendroglial cell line, OLN-93, as previously described $^{29}$ and kindly provided by Jonathan Gilthorpe (Department of Integrative Medical Biology, Umeå University).

Vesicle Preparation. All liposomes were prepared using the lipid hydration and extrusion method. Appropriate amounts of lipids dissolved in chloroform were added to a round-bottom flask. The chloroform was first evaporated under a constant airflow while rotating the flask at an angle to form a homogeneous lipid film on the bottom of the flask. The flask was then left under airflow for $1.5 \mathrm{~h}$ to ensure that all of the chloroform evaporates. After drying, the lipid film was hydrated with HEPES-buffered saline (HBS), consisting of $10 \mathrm{mM}$ HEPES and $150 \mathrm{mM} \mathrm{NaCl}$, with $\mathrm{pH}=7.4$ and extruded using a mini extruder (Avanti Polar Lipids Inc.). The $100 \%$ POPC liposomes used for illustrating the sizeindependence of the FRET-assay were prepared by first freeze-thawing the vesicle solution five times in liquid nitrogen. The liposome solution was then extruded either 31 times through a $400 \mathrm{~nm}$ polycarbonate membrane or 11 times through a $100 \mathrm{~nm}$ membrane followed by 31 times through a $30 \mathrm{~nm}$ membrane. All other vesicles were extruded 11 times through a polycarbonate membrane with $100 \mathrm{~nm}$ pores without freeze-thawing cycling. The FRET liposomes were composed of $99 \mathrm{~mol} \%$ POPC, $0.5 \mathrm{~mol} \%$ Rhod-PE, and 0.5 mol \% NBD-PE. The liposomes used to establish the calibration curve were made of $100 \%$ POPC.

Phosphorus Assay. To quantify the phospholipid content of the liposome stocks prepared as described above and to account for possible lipid losses during the extrusion procedure, a phosphorus assay was used. The assay is based on the molybdenum blue method ${ }^{30,31}$ and was performed as described by Paraskova et al. ${ }^{32}$ with the modification that digestion was performed solely by dry ashing for at least $4 \mathrm{~h}$ at $550{ }^{\circ} \mathrm{C}$. Calcination was performed in a Carbolite CWF 1200 furnace (Carbolite Gero Limited, Hope Valley, U.K.). Absorbance at $882 \mathrm{~nm}$ was measured three times to obtain an average in the multimode microplate reader Varioskan Flash (Thermo Fisher Scientific). The band width was $5 \mathrm{~nm}$, and the measurement time was $100 \mathrm{~ms}$. The calibration curves for the assay were established using a $0.65 \mathrm{mM}$ phosphorus standard solution (Sigma-Aldrich). The calibration curve was established without calcinating the standard solution, as a 
preliminary test showed no difference in the absorbance between calcinated and uncalcinated standard solutions (data not shown).

Sonication Procedure for Lipid Mixing. A bath sonicator (ultrasonic frequency: $37 \mathrm{kHz}$; ultrasonic power (effective/max): 140/560 W; Elmasonic S40H, Germany) was used to induce vesicle fusion. Unless stated otherwise, the FRET assay was performed as follows: FRET liposomes and either calibration liposomes or sample vesicles were mixed together keeping the concentration of FRET liposomes constant at $4.3 \times 10^{-8} \mathrm{~mol} / \mathrm{mL}$ of lipids $(1 \mu \mathrm{L}$ of $1 \mathrm{mg} / \mathrm{mL}$ added), corresponding to a surface area concentration (SAC) of $82.5 \times 10^{14} \mathrm{~nm}^{2} / \mathrm{mL}$ of FRET liposomes, while the concentration of sample vesicles was varied accordingly to bring the mixture to a total of $60 \mu \mathrm{L}$. This vesicle mixture was sonicated in a prewarmed, degassed, and half-full Elmasonic $\mathrm{S} 40 \mathrm{H}$ bath sonicator making sure to place the sample in vibrational "hotspots", where the vibrations are the strongest. Water temperature was kept constant $\left( \pm 1{ }^{\circ} \mathrm{C}\right)$ using small additions of ice.

Spectrofluorimetry. For fluorescence measurements, 50 $\mu \mathrm{L}$ of the sonicated samples was mixed with $50 \mu \mathrm{L}$ of buffer in the well of a black-bottomed NUNC plate (634-0006, Thermo Scientific) and scanned for fluorescence spectra with the multimode microplate reader Varioskan Flash (Thermo Fisher Scientific). To acquire an emission spectrum, the sample was excited at $460 \mathrm{~nm}$ with an emission scan between 490 and 650 $\mathrm{nm}$. The excitation bandwidth was $12 \mathrm{~nm}$, and the dynamic range was set to "Medium Low". The measurement time was $100 \mathrm{~ms}$. In all measurements, the background signal of pure buffer was subtracted from the FRET signal. Rhodamine and NBD have listed spectral intensity peaks at 583 and $535 \mathrm{~nm}$, respectively. However, we observed the intensity peaks at 588 $\mathrm{nm}$ for rhodamine and $535 \mathrm{~nm}$ for NBD. These wavelengths were therefore used to estimate the respective peak intensity values and to calculate the FRET signal (ratio of the rhodamine and NBD peaks).

Nanoparticle Tracking Analysis. Nanoparticle tracking analysis (NTA) measurements were performed with a ZetaView PMX 110 NTA device from Particle Metrix $\mathrm{GmbH}$ (Inning am Ammersee, Germany). The device was calibrated with $102 \mathrm{~nm}$ standard beads from the manufacturer before measurements. "Sensitivity" and "Shutter" parameters were determined to have optimal values of "Sensitivity" $=75$ and "Shutter" $=35$, based on manufacturer's guidelines and after testing different parameters on the POPC vesicle sample. Samples were diluted to an optimal concentration for analysis (between 100 and 200 visible particles), and $\sim 800 \mu \mathrm{L}$ of the sample was injected into the measurement chamber and measured. The device performs measurements at 11 different positions in the measurement chamber and gives an average as a result. Each vesicle sample was measured in triplicate to obtain an average.

Dynamic Light Scattering. Dynamic light scattering (DLS) being more sensitive for the detection of vesicles with diameters below $50 \mathrm{~nm},{ }^{20}$ NTA data was complemented with DLS measurements. These measurements were performed with a ZetaSizer Nano-s (Malvern Instruments, U.K.). Each sample is scanned at least 10 times, and the results were averaged to present the size profile. Each vesicle sample was measured in triplicate to obtain an average.

\section{RESULTS AND DISCUSSION}

Establishing a Calibration Curve. To take advantage of the FRET assay presented in Figure 1, our approach is based on first establishing a calibration curve using a vesicle sample standard of known concentration and on relating the change in the fluorescence spectrum of the FRET vesicles to the total surface area of the sample vesicles. The total surface area of the sample is reported as the total surface area fraction of the sample (called sample fraction here) and is defined as

$$
\text { sample fraction } \equiv \frac{\mathrm{TSA}_{\text {sample }}}{\mathrm{TSA}_{\mathrm{FRET}}+\mathrm{TSA}_{\text {sample }}}
$$

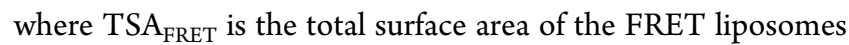
and $\mathrm{TSA}_{\text {sample }}$ is the total surface area of the sample vesicles. In this case, the FRET signal is reported as the fluorescence intensity of the emission spectra at $\lambda_{\mathrm{A}}=588 \mathrm{~nm}$ divided by the fluorescence intensity at $\lambda_{\mathrm{D}}=535 \mathrm{~nm}$, where the wavelengths are the emission peaks for rhodamine and NBD, respectively. As further detailed in Supporting Information Section 1, the change of the FRET signal as a function of the sample fraction ( $x$ in eq 2) can be predicted theoretically by assuming negligible FRET between neighboring particles in the solution and negligible influence of liposome curvature on the FRET signal. Under these assumptions, the system can be approximated as a flat $2 \mathrm{D}$ surface and using the analytical solution for the relative quantum yield proposed by Wolber and Hudson. ${ }^{33}$ The FRET signal is then described by

$$
\begin{aligned}
\text { FRET signal } \equiv & \frac{I(A)}{I(D)} \\
= & Y_{1}\left(-1+\frac{1}{A_{1} \mathrm{e}^{-k_{1} C_{0}(1-x)}+A_{2} \mathrm{e}^{-k_{2} C_{0}(1-x)}}\right) \\
& +Y_{2}
\end{aligned}
$$

In eq $2, A_{1}, A_{2}, k_{1}$, and $k_{2}$ are system independent coefficients that have been determined numerically by Wolber and Hudson; ${ }^{33,34} Y_{1}$ and $Y_{2}$ are the constants related to the spectral properties of donor and acceptor fluorophores (see SI Section 1 for details), $x$ is the sample fraction, and $C_{0}$ is defined as

$$
C_{0} \equiv R_{0}{ }^{2} \mathcal{c}
$$

where $R_{0}$ is the Förster length of the fluorophore pair and $c$ is the number of acceptor fluorophores per unit area.

In this work, pure POPC vesicles were chosen as the standard for calibration curve production. This choice was motivated by the fact that phosphocholine lipids with asymmetric acyl chains are both the most common lipid in eukaryotic membranes ${ }^{35}$ and frequently used in the creation of artificial model membranes. Additionally, POPC and the dyelipid conjugates (NBD-PE and Rho-PE) are all phospholipids whose concentrations in the final working solutions could be accurately quantified orthogonally using the molybdenum blue method for phosphorus quantification, ${ }^{32}$ thereby making it possible to account for the potential material loss during vesicle preparation and thus to ensure that the established calibration curve was accurate. The acceptor fluorophore content in the FRET liposomes was set to $0.5 \mathrm{~mol} \%$, i.e., in the range where the change in FRET efficiency is most sensitive to changes in acceptor surface density. ${ }^{23}$ 
The proposed assay strategy relies on the assumption that the maximum mixing between sample vesicles and FRET liposomes is achieved. We therefore assessed the effect of different sonication parameters on vesicle fusion efficiency and chose a combination of sonication time and temperature that ensures maximum fusion. It was determined that a minimum of 20 min of sonication time was needed for full mixing to occur at $40{ }^{\circ} \mathrm{C}$ (Figure $2 \mathrm{a}$ ). Conversely, the ability to fuse the vesicles

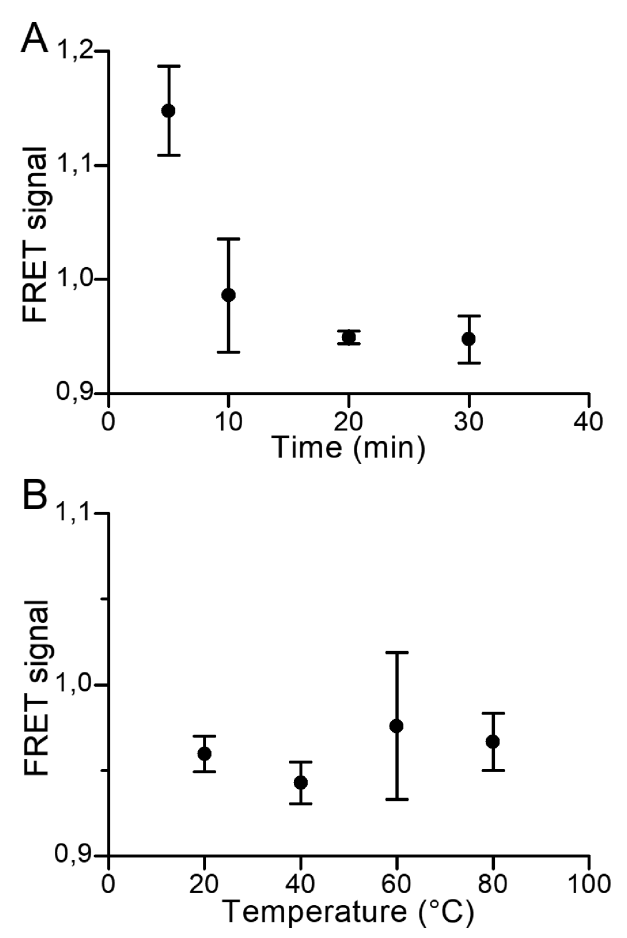

Figure 2. Influence of sonication parameters on FRET signals. (A) FRET signal as a function of sonication time with sonication temperature fixed at $40{ }^{\circ} \mathrm{C}$. (B) FRET signal as a function of sonication temperature, with sonication time fixed at $20 \mathrm{~min}$. FRET signals are the average of three spectra obtained in independent experiments, with standard deviation as the error.

via bath sonication showed no dependency on temperature between 20 and $80^{\circ} \mathrm{C}$ (Figure 2b), indicating that a calibration curve established at a given temperature is valid over a broad range of sonication temperatures. Control experiments confirmed that changes in the FRET signal upon sonication were not an artifact of fluorophore damage (Figure S1). Thus, the calibration curves used in this work were obtained by sonication at $40{ }^{\circ} \mathrm{C}$ for $20 \mathrm{~min}$.

A standard calibration curve displayed as the FRET signal versus the sample fraction is shown in Figure 3A. The experimental data was in excellent agreement with the behavior predicted by the theoretical model, as shown by fitting the data with eq 3 using a $C_{0}$ value of 0.35 estimated from an acceptor surface density value of 0.35 molecules $/ \mathrm{nm}^{2}$, corresponding to a rhodamine lipid concentration of $0.5 \mathrm{~mol} \%$ and estimated using an area-per-lipid of $0.63 \mathrm{~nm}^{236}$ and a Förster length $\left(R_{0}\right)$ of $6.6 \mathrm{~nm} .{ }^{37}$ Additionally, the behavior for sample fraction values between 0.2 and 0.9 can be approximated with a linear fit, making this curve easily usable as a calibration curve within this range.

Using FRET liposomes (99 mol \% POPC) and pure POPC calibration vesicles, whose exact concentrations have been verified using the phosphorous assay, it is possible to use
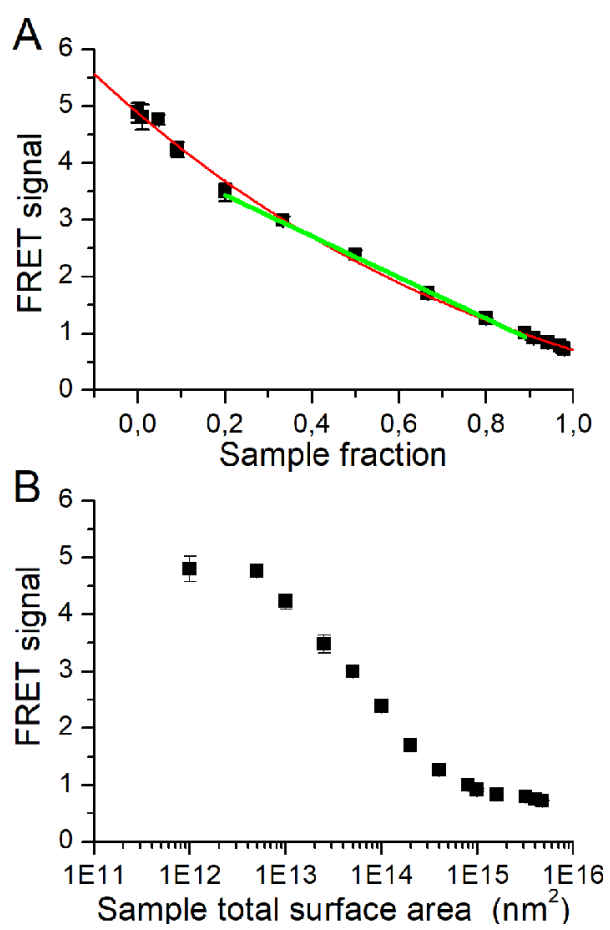

Figure 3. Calibration curve established with POPC calibration vesicles of known concentration. (A) Calibration curve represented as a FRET signal (fluorescence intensity of the emission spectra at 588 $\mathrm{nm}$ divided by the fluorescence intensity at $535 \mathrm{~nm}$ ) versus sample fraction (surface area fraction of the calibration vesicles). The data can be fitted with expression (3) (fit shown in red). Using the following coefficients $A_{1}=0.6463, A_{2}=0.3537, k_{1}=4.7497$, and $k_{2}=$ 2.0618 as proposed in reference 33 for the case where the closest distance between the donor and acceptor $\left(R_{\mathrm{e}}\right)$ is much smaller than the Förster length $\left(R_{0}\right)$ i.e., for $R_{\mathrm{e}} / R_{0}=0, C_{0}=0.35$ as estimated from eq 3 for the FRET vesicles used here. Based on the best fit, $Y_{1}=1.78$ and $Y_{2}=0.71$. Additionally, the data can be fitter linearly for fractions ranging from 0.2 to 0.9 (fit shown in green; $R^{2}$ value: 0.99659 ). (B) Calibration curve represented as the FRET signal versus total surface area (TSA) of the POPC calibration vesicles. All data points are the averages of three measurements, with standard deviations as error bars.

POPC's area per lipid $\left(0.63 \mathrm{~nm}^{236}\right)$ to calculate both TSA $\mathrm{FRET}$ and $\mathrm{TSA}_{\text {sample, }}$, respectively, allowing the calibration curve to be expressed directly as a function of the sample vesicle's TSA (Figure 3B). A representation on a logarithmic scale yields a sigmoidal behavior, clearly illustrating that for the chosen FRET liposome concentration used in this work the assay is best-suited to quantify samples within a $\mathrm{TSA}_{\text {sample }}$ range of $\sim 5$ $\times 10^{12}$ to $4 \times 10^{14} \mathrm{~nm}^{2}$, i.e., within over 2 orders of magnitude surface area. Additionally, the sigmoidal plot allows the limit of detection (LOD) of the assay to be more easily determined; the LOD, i.e., the lowest quantity of analyte that can be distinguished from the absence of it, was defined as the $\mathrm{TSA}_{\text {sample }}$ value with a signal corresponding to the mean FRET signal of a blank sample (negative control, FRET liposomes sonicated alone in the buffer of interest) minus the 3 -fold standard deviation of three independent measurements of the blank signal. In our case, the LOD was found to be $9.5 \times 10^{12}$ $\mathrm{nm}^{2}$, which equates to $1.18 \times 10^{8}$ vesicles, as estimated from simple geometrical considerations, assuming an average vesicle diameter of $160 \mathrm{~nm}$ (measured by DLS; Figure S2) and an area-per-lipid value of $0.65 \mathrm{~nm}^{2} .^{36}$ As the protocol is based on $60 \mu \mathrm{L}$ of the sample solution, the LOD would correspond to a 
surface area concentration (SAC) of $1.64 \times 10^{14} \mathrm{~nm}^{2} / \mathrm{mL}$ or roughly $2.03 \times 10^{9}$ vesicles $/ \mathrm{mL}$ (for vesicles with a diameter of $160 \mathrm{~nm}$ ). It should be noted that the LOD value does not depend on the type of material analyzed but is rather dependent on the blank signal, resulting from sonication of FRET vesicles and sample-free background solution. It is therefore important to generate a calibration curve in the appropriate background since autofluorescence, residual lipids, or even free proteins may contribute to altering the background signal (see SI Section 7 for more discussion).

To investigate if the performance of the assay is dependent on vesicle size, two vesicle batches of distinct mean diameters, i.e., mean diameters of $79 \pm 0.4$ and $301 \pm 10 \mathrm{~nm}$, were produced. Their size distributions were measured with DLS (Figure S2). At equal lipid concentration (as determined by the phosphorus assay), the emission spectra of the fused vesicles were indistinguishable (Figure 4), confirming the size independence of the assay.

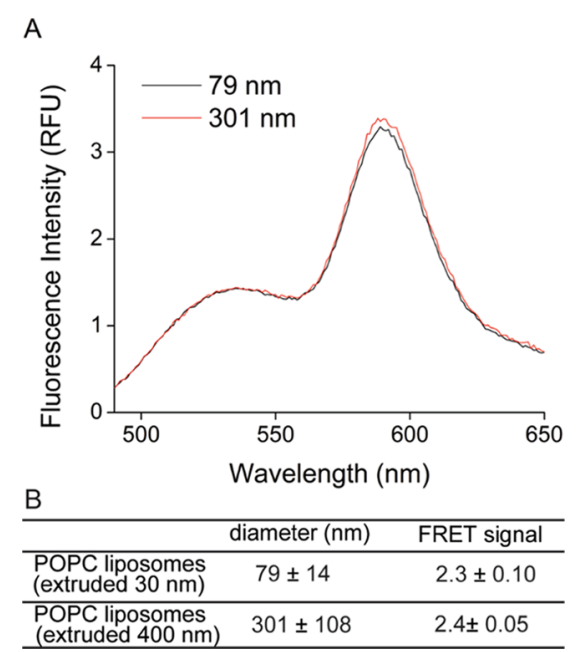

Figure 4. (A) Emission spectrum after sonication (excitation wavelength: $460 \mathrm{~nm}$ ) for POPC vesicles of mean diameters of 79 $\mathrm{nm}$ (black curve) and $301 \mathrm{~nm}$ (red curve). The curve was obtained by sonicating $2 \mu \mathrm{g}$ of sample POPC vesicles with $1 \mu \mathrm{g}$ of FRET vesicles. (B) Average and standard deviation of the FRET signal for three measurements. The diameter values are the averages of three independent size measurements. Error bars for the diameter values are the standard deviations of the distribution.

Quantification of Various Vesicle Species Using the FRET Assay and Nanoparticle Tracking Analysis. After having established the dependence between the FRET signal and TSA of the sample vesicles, we demonstrated the assay's potential for the quantification of vesicle species of complex composition. Specifically, samples containing extracellular vesicles from eukaryotic cells (EVs), outer membrane vesicles (OMVs), and the enveloped virus herpes simplex virus-2 (HSV-2) were used. The results were further compared with NTA-based quantification. Benchmarking of the FRET-based assay against NTA is particularly meaningful since the latter technique allows for the determination of size distributions by looking at particles one-by-one, which can easily be converted into a SAC using the equation to calculate the surface area of a sphere.

For each sample, fusion was verified by sonicating different sample volumes. The effect of sonication temperature was investigated between 20 and $80{ }^{\circ} \mathrm{C}$ (Figure S3). As no statistically relevant trend was observed between temperature and degree of mixing, all samples were sonicated at $40{ }^{\circ} \mathrm{C}$. Table 1 provides a summary of the various samples' SACs obtained using the FRET assay, mean particle diameter, NTA quantification in particles $/ \mathrm{mL}$, and the conversion of the NTA quantification data into terms of SAC, for comparison. For comparison, the FRET-assay and NTA values obtained from POPC liposomes are also included. The calibration curve used for quantification in this case can be found in Figure S4, while size distribution profiles measured with NTA are shown in Figure S5.

As evident in Table 1 under the FRET/NTA ratio, our results indicate that the FRET assay yielded larger SAC values than NTA. The difference in the obtained SAC value for both techniques was particularly striking for the POPC liposomes of small diameter (extruded with a $30 \mathrm{~nm}$ pore size), where 40 times more membrane material was detected with the FRET assay, most likely reflecting the limited ability of NTA to detect and track vesicles with diameters below 50-70 nm. ${ }^{19}$ Indeed, it is also important to note that the concentrations of the two POPC samples in Table 1 were matched using the phosphorus assay and that while the FRET assay delivered SACs with good agreement $\left(49.5 \pm 4.5\right.$ vs $\left.53.6 \pm 6.0 \mathrm{~nm}^{2} / \mathrm{mL} \times 10^{16}\right)$, the NTA delivered SACs that differed by an order of magnitude $\left(12.7 \pm 0.6\right.$ vs $\left.1.35 \pm 0.28 \mathrm{~nm}^{2} / \mathrm{mL} \times 10^{16}\right)$. This further illustrates NTA's limited ability to accurately quantify vesicle samples containing large fractions of smaller vesicles.

To gain insights into the precision of the assay, we performed three independent measurements on each sample and estimated the standard deviation and coefficient of variation $(\mathrm{CV}=$ standard deviation/mean $)$. For the FRET

Table 1. Sample Quantification Using the FRET Assay and Nanoparticle Tracking Analysis (NTA) ${ }^{a}$

\begin{tabular}{|c|c|c|c|c|c|c|c|}
\hline & \multicolumn{2}{|l|}{ FRET assay } & \multicolumn{4}{|c|}{ NTA } & \multirow[b]{2}{*}{$\begin{array}{l}\text { FRET } / \mathrm{NTA} \\
\text { ratio }\end{array}$} \\
\hline & $\begin{array}{c}\text { SAC } \\
\left(\mathrm{nm}^{2} / \mathrm{mL} \times 10^{16}\right)\end{array}$ & $\begin{array}{l}\mathrm{CV} \\
(\%)\end{array}$ & $\underset{(\mathrm{nm})}{\operatorname{diameter}}$ & $\begin{array}{c}\text { concentration } \\
\left(\text { particles } / \mathrm{mL} \times 10^{11}\right)\end{array}$ & $\begin{array}{c}\text { SAC } \\
\left(\mathrm{nm}^{2} / \mathrm{mL} \times 10^{16}\right)\end{array}$ & $\begin{array}{l}\mathrm{CV} \\
(\%)\end{array}$ & \\
\hline $\begin{array}{l}\text { POPC liposomes } \\
\text { (extruded with } 100 \mathrm{~nm} \text { filter) }\end{array}$ & $49.5 \pm 4.5$ & 9 & $124 \pm 52$ & $19.8 \pm 0.12$ & $12.7 \pm 0.6$ & 4.9 & 3.9 \\
\hline $\begin{array}{l}\text { POPC liposomes } \\
\text { (extruded with } 30 \mathrm{~nm} \text { filter) }\end{array}$ & $53.6 \pm 6.0$ & 11 & $70 \pm 30$ & $6.6 \pm 1.3$ & $1.35 \pm 0.28$ & 21 & 39.4 \\
\hline OMV's & $7.22 \pm 0.9$ & 12 & $123 \pm 49$ & $1.9 \pm 0.3$ & $1.2 \pm 0.1$ & 8.5 & 6.1 \\
\hline HSV-2 & $2.21 \pm 0.15$ & 6.7 & $175 \pm 86$ & $0.451 \pm 0.092$ & $0.58 \pm 0.23$ & 40 & 3.8 \\
\hline EV's & $21.6 \pm 1.8$ & 8.3 & $126 \pm 59$ & $9.2 \pm 2.3$ & $6.3 \pm 2.0$ & 31 & 3.4 \\
\hline
\end{tabular}

${ }^{a} \pm$ values are the standard deviation of three independent measurements, except for \pm values for the diameter that are the average of the standard deviations of the distribution of three independent measurements. 
assay, the average $\mathrm{CV}$ for vesicle quantification, all samples combined, was $9.4 \pm 2.1 \%$. Such errors are similar to the ones reported in the literature with NTA; ${ }^{38}$ however, in our hands, CVs calculated for the NTA measurements of the different samples showed a quite wide variation with the $100 \mathrm{~nm}$ liposomes providing the best reproducibility and the complex vesicles exhibiting a higher variance.

As with any assay, it is important to know what the limitations are in regard to contaminants that may be present in the sample and how they might perturb the measurement. As this assay is based on vesicles, residual detergents in a sample should be minimized. The presence of free protein (e.g., BSA) was found to affect the assay by interfering with the FRET ratio (Figure S6A); however, as most protocols for EV, OMV, and virus isolation seek to minimize the amount of residual free protein contamination in the sample, this effect is most likely not significant in most cases. However, it is important to gauge the effect of density gradient mediums such as sucrose and Optiprep or of the commonly used cryoprotectant glycerol. Neither sucrose, Optiprep, nor glycerol were found to alter the assay's ability to quantify a vesicle sample (Figure S6A).

If significant levels of free protein are believed to be in a sample or any other contaminant that is feared could interfere with the quantification, then the creation of a calibration curve using a background diluent that contains the suspected contaminants is of course the best strategy to take the effects of those contaminants into account. Additionally, it is advisable to always measure an unknown sample at two different dilutions that fall within the calibration curve range to verify that the sample does not show signs of interfering contaminants.

\section{CONCLUSIONS}

In this work, we have developed and validated a FRET-based method to quantify the total surface area of membranes in a purified vesicle sample. This method relies on altering the emission spectrum of a fluorophore pair subject to FRET through the sonication-induced fusion of vesicles containing a FRET pair and nonlabeled sample vesicles. An advantage of this assay is that it can be performed without any specialized instrumentation; just a relatively cheap bath sonicator and a spectrofluorometer, generic instruments commonly found in biochemistry labs, are sufficient for its execution. As predicted theoretically, the FRET signal is dependent on the sample fraction, making it possible to quantify membrane surface area in a sample over several orders of magnitude after establishing a calibration curve. With the experimental parameters used here, and without extensive assay optimization, we were able to achieve a limit of detection of approximately $10^{8}$ particles $\left(10^{9}\right.$ particles $/ \mathrm{mL})$; a lower LOD could potentially be obtained using a lower concentration of FRET liposomes, thereby shifting the dynamic range of the assay, the limiting factor being the sensitivity of the spectrofluorometer. Additionally, the performance of the assay could be further improved by choosing an optimal fluorophore pair. Our theoretical model suggests that the method sensitivity can be improved by maximizing the value of $C_{0}$ and $Y_{1}$, yielding the steepest decay. This can be achieved by choosing a FRET pair with a larger Förster radius $\left(R_{0}\right)$ and where the acceptor has a high quantum yield and with a narrow emission spectrum.

The assay performance was further compared to that of nanoparticle tracking analysis, a well-established method for particle number quantification and size determination, ${ }^{16}$ making a comparison of surface area concentrations particularly straightforward. A major advantage of our assay as compared to methods relying on the imaging of individual vesicles in scattering mode, such as NTA, is that its performance is independent of vesicle size, making it very well-suited to characterize polydisperse samples containing small vesicles $(<70 \mathrm{~nm})$. Indeed, it has been previously reported that the visualization of lipid vesicles using NTA devices is particularly challenging due to the vesicle's weak scattering properties in aqueous solution, typically limiting detection to vesicles larger than $\sim 50 \mathrm{~nm}$ in diameter. ${ }^{19}$ Our assay was also found to be somewhat superior to NTA in terms of dynamic range, as it allows for direct quantification for vesicle concentrations spanning over 2 orders of magnitude, as compared to the rather limited dynamic range of some NTA instruments (less than 1 order of magnitude). ${ }^{21,39-41}$

In terms of the lowest concentration that can be quantified, we report here an LOD value of $\sim 10^{9}$ particles $/ \mathrm{mL}$, i.e., $1-2$ orders of magnitude higher than that reported in the literature for ZetaView NTA measurements of vesicle samples ${ }^{39,40}$ although it cannot be excluded that these concentrations are underestimated, in the likely case that the smallest vesicles in the population are too small to be detected. Since our assay is size-independent, it can therefore be said that in terms of both LOD and the total amount of material needed to carry out a lipid vesicle quantification, the performance of both techniques is roughly comparable. It should however further be highlighted that the FRET-based assay can be carried out at much lower sample volumes (with the protocol presented here, 58 $\mu \mathrm{L}$ of the sample is sufficient), while according to the provider's specifications, $500 \mu \mathrm{L}$ of samples is needed to carry out an accurate quantification with the ZetaView, ${ }^{42}$ making the FRET-based assay of advantage when low amounts of starting material are available.

In spite of the fact that vesicles of complex composition, from both biological and synthetic origins, are associated with such a broad spectrum of research areas, methods to quantify lipid vesicle concentrations in a robust and versatile manner are surprisingly underdeveloped. While expressing the concentration of a vesicle sample in terms of SAC is unconventional, this measure provides a competitive alternative or complement to expressing vesicle concentration in number of particles/milliliter. Although this assay cannot decouple size from particle numbers, as is the case for NTA, it provides an unambiguous and size-independent quantification of the total amount of membrane material present in a sample. This may be of advantage when studying the biological effect of different EV batches or the efficiency of a liposome-based drug delivery system, where dosing the same total amount of material may be preferred to dosing the same number of particles, which may have significantly different size distributions. Also, it should be noted that this method is unspecific, in that it does not distinguish vesicle subpopulations according to any biomolecular identity (i.e., the presence of a specific marker on its surface or within its lumen). Rather, it relies on vesicle purification prior to analysis. However, the assay's ability to better quantify subpopulations of vesicles with diameters below $100 \mathrm{~nm}$ attained from purification protocols including size exclusion chromatography, differential centrifugation, or affinity chromatography to select vesicles of desired size, density, or composition is likely to make it a powerful quantitative tool in a variety of contexts. 


\section{ASSOCIATED CONTENT}

\section{SI Supporting Information}

The Supporting Information is available free of charge at https://pubs.acs.org/doi/10.1021/acs.analchem.0c02271.

Theoretical model describing the behavior of the FRET signal in the FRET assay; additional figure showing the effect of sonication parameters on FRET-vesicle fluorophores; calibration curve and additional figures illustrating parameter optimization for the quantification of EV, OMV, and HSV samples; size distributions of vesicles as measured with DLS and NTA; and additional data showing the effect of contaminants on the assay (PDF)

\section{AUTHOR INFORMATION}

\section{Corresponding Authors}

Hudson Pace - Section of Virology, Department of Clinical Microbiology, Wallenberg Centre for Molecular Medicine, and Department of Integrative Medical Biology, Umeå University, 90185 Umeå, Sweden; 이이.org/0000-0001-51162577; Email: hudson.pace@umu.se

Marta Bally - Section of Virology, Department of Clinical Microbiology and Wallenberg Centre for Molecular Medicine, Umeå University, 90185 Umeå, Sweden; ํㅏㅇㅣ.org/ 0000-0002-5865-8302; Email: marta.bally@umu.se

\section{Authors}

Konrad Thorsteinsson - Section of Virology, Department of Clinical Microbiology and Wallenberg Centre for Molecular Medicine, Umeå University, 90185 Umeå, Sweden

Erik Olsén - Division of Nano and Biophysics, Department of Physics, Chalmers University of Technology, 41296 Gothenburg, Sweden; (1) orcid.org/0000-0002-4002-0917

Eneas Schmidt - Division of Nano and Biophysics, Department of Physics, Chalmers University of Technology, 41296 Gothenburg, Sweden

Complete contact information is available at:

https://pubs.acs.org/10.1021/acs.analchem.0c02271

\section{Author Contributions}

${ }^{\perp}$ H.P. and M.B. contributed equally to this work.

\section{Notes}

The authors declare no competing financial interest.

\section{ACKNOWLEDGMENTS}

This work was supported by the Swedish Research Council under Grant 2017-04029 and the Knut and Alice Wallenberg Foundation. The contribution of the following people is further acknowledged: Nadia Peerboom (Department of Physics, Chalmers University of Technology) for assistance with setting up the experiments; Karin Norling (Department of Physics, Chalmers University of Technology) for help with phosphorus quantification; Madeleine Ramstedt (Department of Chemistry, Umeå University) for providing the outer membrane vesicles; Jonathan Gilthorpe and Birendra Singh (Department of Integrative Medical Biology, Umeå University) for providing the extracellular vesicle sample; and Tomas Bergström (Department of Infectious Medicine, Gothenburg University) for providing herpes simplex virus samples.

\section{REFERENCES}

(1) Raposo, G.; Nijman, H. W.; Stoorvogel, W.; Leijendekker, R.; Harding, C. V.; Melief, C. J. M.; Geuze, H. J. J. Exp. Med. 1996, 183, $1161-1172$.

(2) Théry, C.; Ostrowski, M.; Segura, E. Nat. Rev. Immunol. 2009, 9, 581-593.

(3) Bobrie, A.; Colombo, M.; Raposo, G.; Thery, C. Traffic 2011, 12, 1659-1668.

(4) Admyre, C.; Telemo, E.; Almqvist, N.; Lotvall, J.; Lahesmaa, R.; Scheynius, A.; Gabrielsson, S. Allergy 2008, 63, 404-408.

(5) Alexander, M.; Hu, R.; Runtsch, M. C.; Kagele, D. A.; Mosbruger, T. L.; Tolmachova, T.; Seabra, M. C.; Round, J. L.; Ward, D. M.; O'Connell, R. M. Nat. Commun. 2015, 6, No. 7321.

(6) Buzas, E. I.; Gyorgy, B.; Nagy, G.; Falus, A.; Gay, S. Nat. Rev. Rheumatol. 2014, 10, 356-364.

(7) Lamichhane, T. N.; Sokic, S.; Schardt, J. S.; Raiker, R. S.; Lin, J. W.; Jay, S. M. Tissue Eng., Part B 2015, 21, 45-54.

(8) Nakamura, Y.; Miyaki, S.; Ishitobi, H.; Matsuyama, S.; Nakasa, T.; Kamei, N.; Akimoto, T.; Higashi, Y.; Ochi, M. FEBS Lett. 2015, $589,1257-1265$.

(9) Al-Nedawi, K.; Meehan, B.; Rak, J. Cell Cycle 2009, 8, 20142018.

(10) Candelario, K. M.; Steindler, D. A. Trends Mol. Med. 2014, 20, 368-374.

(11) Schneider, A.; Simons, M. Cell Tissue Res. 2013, 352, 33-47.

(12) Kaparakis-Liaskos, M.; Ferrero, R. L. Nat. Rev. Immunol. 2015, $15,375-387$.

(13) Allen, T. M.; Cullis, P. R. Adv. Drug Delivery Rev. 2013, 65, 3648.

(14) Gregoriadis, G.; Wills, E. J.; Swain, C. P.; Tavill, A. S. Lancet 1974, 303, 1313-1316.

(15) Torchilin, V. P. Nat. Rev. Drug Discovery 2005, 4, 145-160.

(16) Rupert, D. L.; Claudio, V.; Lasser, C.; Bally, M. Biochim. Biophys. Acta 2017, 1861, 3164-3179.

(17) Heider, S.; Metzner, C. Virology 2014, 462-463, 199-206.

(18) Malloy, A.; Carr, B. Part. Part. Syst. Charact. 2006, 23, 197204

(19) van der Pol, E.; Coumans, F. A.; Grootemaat, A. E.; Gardiner, C.; Sargent, I. L.; Harrison, P.; Sturk, A.; van Leeuwen, T. G.; Nieuwland, R. J. Thromb. Haemostasis 2014, 12, 1182-1192.

(20) van der Pol, E.; Hoekstra, A. G.; Sturk, A.; Otto, C.; van Leeuwen, T. G.; Nieuwland, R. J. Thromb. Haemostasis 2010, 8, 2596-2607.

(21) Gardiner, C.; Ferreira, Y. J.; Dragovic, R. A.; Redman, C. W.; Sargent, I. L. J. Extracell. Vesicles 2013, No. 19671.

(22) Colombo, M.; Moita, C.; van Niel, G.; Kowal, J.; Vigneron, J.; Benaroch, P.; Manel, N.; Moita, L. F.; Thery, C.; Raposo, G. J. Cell Sci. 2013, 126, 5553-5565.

(23) Struck, D. K.; Hoekstra, D.; Pagano, R. E. Biochemistry 1981, 20, 4093-4099.

(24) Marsden, H. R.; Tomatsu, I.; Kros, A. Chem. Soc. Rev. 2011, 40, $1572-1585$

(25) Nakao, R.; Ramstedt, M.; Wai, S. N.; Uhlin, B. E. PLoS One 2012, 7, No. e51241.

(26) Duff, R.; Rapp, F. Nat. New Biol. 1971, 233, 48-50.

(27) Adamiak, B.; Ekblad, M.; Bergstrom, T.; Ferro, V.; Trybala, E. J. Virol. 2007, 81, 13424-13434.

(28) Trybala, E.; Liljeqvist, J. A.; Svennerholm, B.; Bergstrom, T. J. Virol. 2000, 74, 9106-9114.

(29) Muthukrishnan, U. The Release of Histone Proteins from Cells via Extracellular Vesicles. Licentiate Thesis, Comprehensive Summary, Umeå University, Umeå, 2018.

(30) Murphy, J.; Riley, J. P. J. Mar. Biol. Assoc. 1958, 37, 9-14.

(31) Murphy, J.; Riley, J. P. Anal. Chim. Acta 1962, 27, 31-36.

(32) Paraskova, J. V.; Rydin, E.; Sjoberg, P. J. Talanta 2013, 115, 336-341.

(33) Wolber, P. K.; Hudson, B. S. Biophys. J. 1979, 28, 197-210.

(34) Kahya, N.; Schwille, P. J. Fluoresc. 2006, 16, 671-678. 
(35) van Meer, G.; Voelker, D. R.; Feigenson, G. W. Nat. Rev. Mol. Cell Biol. 2008, 9, 112-124.

(36) Kučerka, N.; Nieh, M. P.; Katsaras, J. Biochim. Biophys. Acta, Biomembr. 2011, 1808, 2761-2771.

(37) Wolf, D. E.; Winiski, A. P.; Ting, A. E.; Bocian, K. M.; Pagano, R. E. Biochemistry 1992, 31, 2865-2873.

(38) Bachurski, D.; Schuldner, M.; Nguyen, P. H.; Malz, A.; Reiners, K. S.; Grenzi, P. C.; Babatz, F.; Schauss, A. C.; Hansen, H. P.; Hallek, M.; von Strandmann, E. P. J. Extracell. Vesicles 2019, 8, No. 1596016.

(39) Tian, Y.; Ma, L.; Gong, M.; Su, G.; Zhu, S.; Zhang, W.; Wang, S.; Li, Z.; Chen, C.; Li, L.; Wu, L.; Yan, X. ACS Nano 2018, 12, 671680.

(40) Castoldi, M.; Kordes, C.; Sawitza, I.; Haussinger, D. Sci. Rep. 2016, 6, No. 31869.

(41) Dragovic, R. A.; Gardiner, C.; Brooks, A. S.; Tannetta, D. S.; Ferguson, D. J. P.; Hole, P.; Carr, B.; Redman, C. W. G.; Harris, A. L.; Dobson, P. J.; Harrison, P.; Sargent, I. L. Nanomedicine 2011, 7, 780788.

(42) ZetaView Nanoparticle Analyzer User Manual. Particle Metrix GmbH: Germany. http://www.cnpowder.com.cn/img/product/ 2015/02/docmanual_20150228134242.pdf (accessed July 28, 2020). 